\title{
Rejecting salient distractors: Generalization from experience
}

\author{
Daniel B. Vatterott ${ }^{1} \cdot$ Michael C. Mozer $^{2} \cdot$ Shaun P. Vecera ${ }^{1}$ \\ Published online: 11 December 2017 \\ (C) The Psychonomic Society, Inc. 2017
}

\begin{abstract}
Distraction impairs performance of many important, everyday tasks. Attentional control limits distraction by preferentially selecting important items for limited-capacity cognitive operations. Research in attentional control has typically investigated the degree to which selection of items is stimulus-driven versus goal-driven. Recent work finds that when observers initially learn a task, the selection is based on stimulus-driven factors, but through experience, goal-driven factors have an increasing influence. The modulation of selection by goals has been studied within the paradigm of learned distractor rejection, in which experience over a sequence of trials enables individuals eventually to ignore a perceptually salient distractor. The experiments presented examine whether observers can generalize learned distractor rejection to novel distractors. Observers searched for a target and ignored a salient color-singleton distractor that appeared in half of the trials. In Experiment 1, observers who learned distractor rejection in a variable environment rejected a novel distractor more effectively than observers who learned distractor rejection in a less variable, homogeneous environment, demonstrating that variable, heterogeneous stimulus environments encourage generalizable learned distractor rejection. Experiments 2 and 3 investigated the time course of learned distractor rejection across the experiment and found that after experiencing four color-singleton distractors in different blocks, observers could effectively reject subsequent novel color-singleton distractors. These results suggest that the optimization of attentional control to the task environment can be interpreted as a form of learning, demonstrating experience's critical role in attentional control.
\end{abstract}

Keywords Attentional capture $\cdot$ Cognitive and attentional control $\cdot$ Attention

Although we effortlessly produce appropriate actions when performing simple tasks, such as making a cup of coffee, there are multiple limited-capacity cognitive operations between sensing an item (e.g., the coffee grinder) and producing an appropriate action (e.g., placing coffee beans in the grinder). Effective behavior requires that these limited-capacity operations selectively process relevant items. Attention is the selection of items for these limited-capacity operations, and an important question in attentional research is how limited-capacity mechanisms choose what items to select (i.e., attentional control). Obviously, individuals want to select important items, but what makes an item important? Attentional control research has investigated whether individuals determine an item's importance via visual salience (stimulus-driven inputs) or observers' goals

Shaun P. Vecera

shaun-vecera@uiowa.edu

1 Department of Psychological and Brain Sciences, University of Iowa, W311 Seashore Hall, Iowa City, IA 52242-1407, USA

2 Department of Computer Science, University of Colorado, Boulder, CO 80309-0430, USA (goal-driven inputs; Awh, Theeuwes, \& Belopolsky, 2012; Vecera, Cosman, Vatterott, \& Roper, 2014). Researchers who support the stimulus-driven account claim that individuals initially select the most salient items in the environment (Theeuwes, 2010; Itti \& Koch, 2000). Researchers who support the goal-driven account claim that individuals initially select items that fit the observers' current goals (Folk et al., 1992).

Evidence for the stimulus-driven account primarily derives from the additional-singleton paradigm (Theeuwes, 1992), in which observers search for a shape-singleton target among homogeneously shaped distractors. In half of the trials, the distractors are the same color as the target, but in the other half, one distractor is a different color (i.e., color-singleton distractor). The target is never this oddly colored item, making the color-singleton task irrelevant. Nonetheless, observers respond slower when the color-singleton distractor is present than absent, indicating that the color singleton distracted observers (Theeuwes, 2010). However, a salient but irrelevant color singleton does not always slow responses (Bacon \& Egeth, 1994), suggesting a role for goal-driven factors, such as the search "mode" that a participant might employ, which permits more effective distractor rejection. 
Although researchers typically portray attentional control as an immutable system using either stimulus-driven or goaldriven inputs, recent accounts have emphasized the interplay of stimulus-driven and goal-relevant factors (Gaspelin, Leonard, \& Luck, 2015, 2017). One factor that appears to integrate stimulus- and goal-driven factors is the role that experience plays in the balancing between these inputs (Awh et al., 2012; Vecera et al., 2014). For example, individuals select targets sooner if the targets appear in a consistent, predictable configuration with distractors, an effect termed contextual cuing (Chun, 2000; Chun \& Jiang, 1998). More immediate experience also affects attention: targets are discriminated quicker when preceded by a similar target than a dissimilar target, as in priming of pop out (Maljkovic \& Nakayama, 1994, 1996, 2000; Kristjánsson \& Campana, 2010). The foregoing examples center on how experience influences target localization and discrimination; however, effective attentional selection also requires one to reject distracting information and objects.

Experience appears to affect distractor rejection. For instance, Leber and Egeth (2006) trained observers with displays that either encouraged observers to search specifically for the target (i.e., feature-search mode; Bacon \& Egeth, 1994) or generally for unique items (i.e., singleton-detection mode; Pashler 1988). All observers then completed a test phase with displays, termed "option trials," in which search could be deployed in either feature-search mode or singleton-search mode. Leber and Egeth (2006) found that color singletons during the option trials did not distract observers if the observers previously trained with displays that encouraged them to search for a specific target. Color singletons during the option trials did distract observers if the observers previously trained with displays that encouraged them to search for unique items. Thus, past experience using a nonspecific, singleton search strategy made observers susceptible to distraction.

Vatterott and Vecera (2012) investigated the role of distractorrejection experience on attentional control. Observers began with a training block that encouraged a precise search strategy, searching for a target shape (e.g., circle) among heterogeneously shaped distractors (triangles, diamonds, and squares). During this training phase, color-singleton distractors never appeared. Following the training phase, blocks of trials were presented in which a color-singleton distractor appeared in $50 \%$ of the trials. Importantly, the color of the singleton distractor changed each block. Varying the distractor color allowed Vatterott and Vecera (2012) to distinguish among three alternative mechanisms of attentional control. If search strategy alone was sufficient to reject color-singleton distractors, then the color singletons should never distract individuals. Alternatively, observers might need general experience rejecting any salient distractor. In this case, color singletons in the early part of the first block distract observers, because they are novel, but observers learn to reject them (say, in the second half of the first test block) and then effectively reject all following color-singleton distractors. Finally, observers might require experience rejecting each distinct color singleton to reject other singletons of the same color. In this scenario, each time the color of the singleton changes, the color-singleton distract observers in the early part of each block before observers have sufficient experience to reject it. Vatterott and Vecera (2012) found support for the final hypothesis: Even when observers use feature-search mode, they must experience each color singleton to learn to reject them effectively. These results suggest that observers learn to reject distractors through item-specific experience. We refer to this item-specific learning as learned distractor rejection.

Recent research demonstrated that attentional control constantly adjusts to the environment (Cosman \& Vecera, 2014; Leber \& Egeth, 2006; Vatterott \& Vecera, 2012), but little is known about how attentional learning compares to other types of learning. Vatterott and Vecera (2012) demonstrated that at least one type of attentional learning - learned distractor rejection-is item-specific. Other forms of learning, such as skill learning, often exhibit high specificity, but this learning generalizes under the right circumstances, for example, when practice is variable (Schmidt \& Bjork, 1992). The experiments in this article examined whether observers can generalize learned distractor rejection. We first investigated whether variable practice encourages generalization of learned distractor rejection. Previously, we found evidence that learned distractor rejection was item-specific when the salient distractors appeared in a blocked manner (Vatterott \& Vecera, 2012). We asked if a more variable, heterogeneous distractor environment could produce a distractor rejection strategy that generalized to novel items.

Previous research in a variety of domains has demonstrated that variable environments help individuals to generalize learning more effectively to novel environments (Schmidt \& Bjork, 1992). For example, practicing bean bag tossing at a variable-distance target produces more accurate tosses during a testing phase than does practicing on a fixed-distance target (Kerr \& Booth, 1978). In a perceptual coincident timing task, Catalano and Kleiner (1984) sat observers in front of lights that illuminated in succession to create the perception of an object moving toward the observer at a constant rate. Observers responded when the light closest to the observer lit. In test trials, observers who were trained with variableapproach speeds generalized better to novel-approach speeds than observers trained with constant-approach speeds. The benefits of variable practice extend to speech perception: When learning to discriminate the English phonemes /1/ and $/ \mathrm{r} /$, native Japanese participants were better able to generalize to new English speakers when those participants had heard the phonemes produced by varied speakers, suggesting that variability in phoneme production across speakers allowed phoneme discrimination to generalize more effectively to a new talker (Lively, et al., 1993). Similarly, category formation is 
influenced by the variability of category members. When category members were highly variable during category learning, participants' transfer to new stimuli was better than if category members were less variable (Homa \& Vosburgh, 1976).

The current experiments test whether a heterogeneous environment is better suited than a homogeneous environment for training observers to reject novel distractors. If learned distractor rejection uses domain general learning mechanisms similar to other types of learning, a variable, heterogeneous distractor environment might enable observers to reject novel distractors without previously experiencing these specific distractors.

The following experiments are largely modeled after Vatterott and Vecera (2012), with some key exceptions. In Experiment 1, half of the observers experienced a heterogeneous stimulus environment in which three different colorsingletons appeared interspersed throughout three blocks (Mixed group). The other half of observers experienced a homogeneous stimulus environment in which a different singleton color appeared in each of three blocks (Blocked group) and is constant within the block, replicating Vatterott and Vecera (2012). As in our previous experiments (Vatterott \& Vecera, 2012), in Experiment 1 we analyzed the Blocked group by collapsing across trials within a training block. This procedure increases the number of trials for analysis, allowing us to examine the effect of a novel distractor when it appears at the beginning of a block of trials versus at the end of that block. Such collapsing is not possible in the mixed blocks, because all distractor colors appear throughout, so we analyzed these trials by each individual training block.

Both the Blocked group and the Mixed group were then tested with a novel distractor color in a final block of trials; this final block always contained a single distractor color, which appeared in half of the trials. This test block provided the critical test of training variability, because this final block was identical for both the Blocked and Mixed groups. If learned distractor rejection is better generalized following variable training than fixed training, then a novel color singleton in the fourth block will initially distract observers in the Blocked group, but it may not distract observers in the Mixed group. If learned distractor rejection is completely item-specific and shows no tendency to generalize, then the novel color singleton will initially distract observers in both groups.

\section{Experiment 1}

\section{Methods}

Participants Thirty-seven University of Iowa undergraduates completed the experiment for partial course credit. All reported normal or corrected-to-normal vision.
Apparatus A Macintosh Mini computer using MATLAB and the Psychophysics Toolbox (Brainard, 1997) collected responses and presented the stimuli on a 17-in. CRT display. Observers sat approximately $60 \mathrm{~cm}$ from the display.

Stimuli and procedure Six colored shapes equally spaced around the circumference of an imaginary circle centered at fixation with a radius of $4.2^{\circ}$ composed the displays. Fixation was a small white circle in the center of the screen. Each shape was roughly $2.5^{\circ}$ square and contained a randomly vertically or horizontally oriented line $\left(0.7^{\circ} \times 1^{\circ}\right)$. The stimuli consisted of a target (circle) and five distractors (triangle, diamond, or square). The target position was chosen pseudo-randomly on each trial. The identity of each distractor was chosen pseudorandomly on each trial, with the only constraint being that the distractors were not all the same identity. The target and all distractors were green (RGB 0, 255,0) except on singleton present trials when one distractor was either red (RGB 255, 0, 0 ), yellow (RGB 255, 255, 0), purple (RGB 255, 0, 255), or orange (RGB $255,150,0){ }^{1}$ The color-singleton position was chosen randomly from among the distractors on each trial.

Each trial began with only the fixation point visible for $1,000 \mathrm{~ms}$ followed by the search display for $5,000 \mathrm{~ms}$ or until response. If an observer failed to respond within $5,000 \mathrm{~ms}$, the observer was encouraged to respond faster and the trial was marked as incorrect. A beep informed observers of incorrect responses.

Observers pressed either the " $\mathrm{z}$ " or " $\mathrm{m}$ " key to indicate the orientation of the line within the target green circle. Key binding was counter-balanced across observers. The orientation of the line within the circle was chosen pseudo-randomly on each trial. Observers were instructed to respond as quickly and accurately as possible and that a differently colored item might appear on some of the trials, but this item would never be the target, so they should do their best to ignore it. Eye movements were not monitored, but observers were encouraged to maintain fixation.

All observers started the experiment with a 60-trial training block in which observers searched for the target circle among heterogeneously shaped green distractors. Following the training block, all observers completed 4 test blocks of 48 trials each. One of the distractors was differently colored (i.e., a color singleton) in $50 \%$ of the trials. The color of the color singleton remained the same throughout an entire test block but changed between blocks in the Blocked group. See the left

\footnotetext{
${ }^{1}$ Our stimuli were not equiluminant, which often is the case in studies of attentional capture. Our distractor colors were chosen to produce similar amounts of distraction, and preliminary analyses of data from many of our experiments finds that the different distractor colors produce qualitatively similar results, namely, more distraction when they are first encountered and effective distractor rejection after those distractors have been encountered for several dozen trials (Vatterott, 2015). Most important, we counterbalanced color and block to ensure that experience effects are not attributable to specific distractor colors.
} 
panel of Fig. 1 for a depiction of this procedure. The same three-colored color singletons appeared intermixed in the first three blocks in the Mixed group. See the right panel of Fig. 1 for a depiction of this procedure. A single, novel, color singleton appeared in 50\% of the trials of the fourth test block. This test block was identical between the Blocked and Mixed groups. The order of the color-singleton colors was counterbalanced across observers. A short, self-paced rest break preceded each block.

\section{Results}

RTs more than 5 standard deviations above and 2 standard deviations below an observer's mean of each condition were removed from the analysis. This trimming eliminated less than $1 \%$ of the data. Incorrect RTs and RTs following an incorrect response also were removed from the analysis. Three observers from the Blocked group and two from the Mixed group were excluded due to accuracy 2.5 standard deviations below the average accuracy of all observers.

For the Blocked group, we compared RTs in the first and second halves of blocks 1-3 for trials in which colorsingletons were present or absent (upper panel of Fig. 2). Blocks 1-3 were collapsed, because we have previously reported that the results are qualitatively similar across these blocks (Vatterott \& Vecera, 2012), and collapsing increases the number of trials for analysis. We split RTs into the first and second halves of blocks, because this convenient analysis adequately measured learned distractor rejection in past work
(Vatterott \& Vecera, 2012). To measure whether singleton distractors distracted Blocked group observers more early in blocks than late, we entered the RT data from blocks 1-3 into a $2 \times 2$ repeated measures ANOVA with trial position (RTs from the first or second half of a block) and singleton presence (singleton present or absent) as the factors. There was no effect of trial position, $F<1$, but we did find a main effect of singleton presence, $F(1,15)=7.75, p<0.05, \eta_{\mathrm{p}}{ }^{2}=0.34$. Longer RTs when the singleton was present $(914 \mathrm{~ms})$ than absent $(877 \mathrm{~ms})$ drove this main effect. The analyses also revealed a marginally significant interaction between trial position and singleton presence, $F(1,15)=3.85, p=0.068, \eta_{\mathrm{p}}{ }^{2}=$ 0.20 . Planned comparisons confirmed that observers responded slower when the color-singleton was present $(937$ $\mathrm{ms})$ than absent $(867 \mathrm{~ms})$ in the first half of blocks, $t(15)=$ $2.84, p<0.05, \mathrm{~d}_{\mathrm{z}}=0.71$, but not the second half, $t<1$ (singleton present: $888 \mathrm{~ms}$; singleton absent: $883 \mathrm{~ms}$ ). Thus, during the first half of blocks color-singletons distracted observers in the Blocked group, replicating Vatterott and Vecera (2012). Color-singletons distracted observers when novel in the first half of blocks but not in the second half when the color-singletons were more familiar.

Because the Mixed group experienced multiple colorsingletons distractors within each block, the block-half analysis that we performed for the Blocked group are not suitable (the distractor statistics did not change across blocks). Instead of a block-half analysis, the lower panel of Fig. 2 shows the Mixed group's RTs as a function of block. To measure changes in distraction across the three blocks, we entered RTs into a

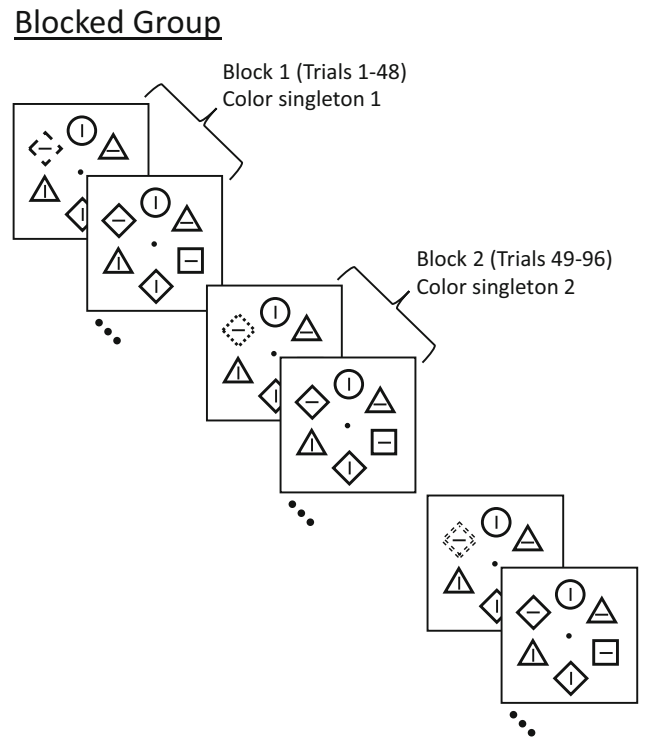

Fig. 1 The left panel depicts the sequence of events for the Experiment 1 Blocked group. A 1,000-ms fixation dot preceded each search display (not pictured). The search display appeared on the screen for 5,000 ms or until response. Color-singleton distractors appeared in $50 \%$ of the trials. The color-singleton's color changed each block. The right panel depicts the sequence of events for the Experiment 1 Mixed group. A

\section{Mixed Group}

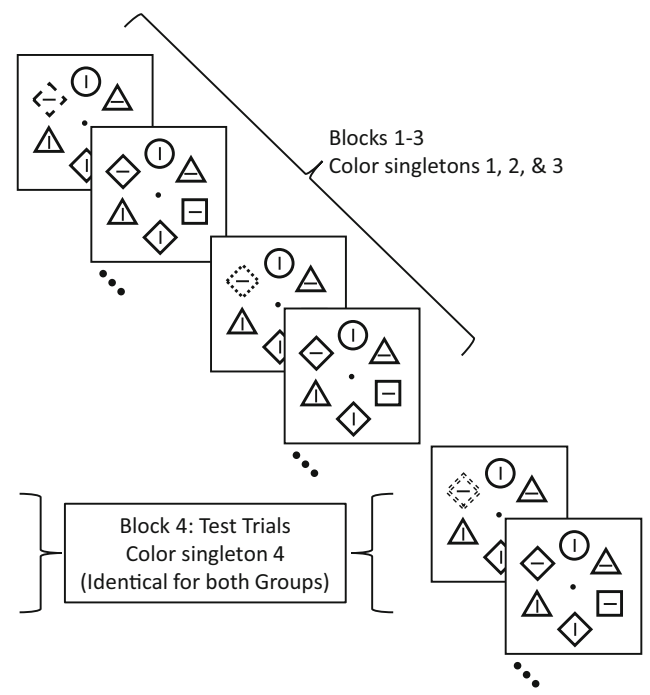

1,000-ms fixation dot preceded each search display (not pictured). The search display appeared on the screen for $5,000 \mathrm{~ms}$ or until response. Color-singletons distractors appeared in $50 \%$ of the trials. Three different color-singletons appeared intermixed within each of the first three blocks. A single novel color-singleton appeared in Block 4 


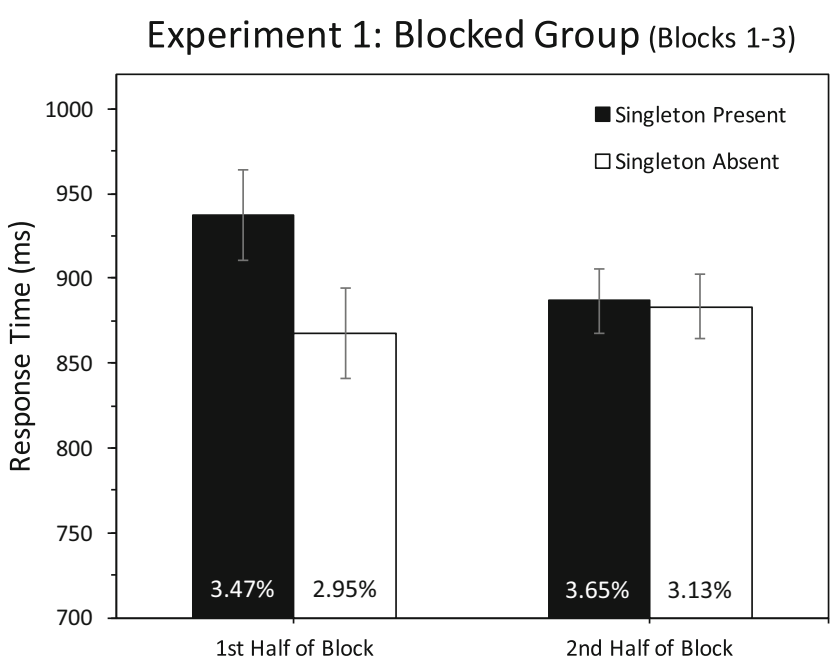

Fig. 2 The left panel depicts Blocked group RTs (in milliseconds) from Blocks 1-3 (averaged) as a function of Trial Position (first half of the block vs. second half of the block) and singleton presence (singleton present vs. singleton absent). The right panel depicts Mixed group RTs

$3 \times 2$ repeated measures ANOVA with the factors block number (blocks 1, 2, and 3) and singleton presence (singleton present vs. absent) as the factors. There was neither a main effect of block number, $F(2,30)=2.03, p>0.14, \eta_{\mathrm{p}}{ }^{2}=0.12$, nor a main effect of singleton presence, $F<1$. The analyses did, however, reveal a significant interaction between block number and singleton presence, $F(2,30)=7.41, p<0.01, \eta_{\mathrm{p}}{ }^{2}$ $=0.33$. Planned comparisons found observers responded slower when the color singleton was present $(841 \mathrm{~ms})$ than absent (786 ms) in the first block, $t(15)=4.66, p<0.01, \mathrm{~d}_{\mathrm{z}}=$ 1.17, but not in the second block, $t<1$ (singleton present: 779 $\mathrm{ms}$; singleton absent: $776 \mathrm{~ms}$ ), or the third, $t(15)=1.71, p>$ $0.1, \mathrm{~d}_{\mathrm{z}}=0.43$ (singleton present: $794 \mathrm{~ms}$; singleton absent: $838 \mathrm{~ms})$. These tests indicate that color singletons distracted observers in the first block when the color singletons were novel, but observers learned to reject the color singletons, and they did not distract observers in the second or third block when the color singletons were more familiar.

Moving onto the critical comparison, we investigated performance in the fourth block of the experiment, which was identical for both groups. In particular, we were interested in whether the stimulus environment changes observers' ability to reject novel color singletons. Inspection of these results, depicted in Fig. 3, indicated that observers in the Blocked group were initially slowed by the new distractor color in block 4 , but these observers quickly tuned their distractor rejection to avoid being slowed by the color singleton. In contrast, observers in the Mixed group showed no initial slowing by the new distractor color in block 4, suggesting these observers had generalized their distractor rejection.

These observations were corroborated by our statistical analyses. Block 4 RTs were entered into a $2 \times 2 \times 2$ mixed measures ANOVA with the factors trial position (first block
Experiment 1: Mixed Group (Blocks 1-3)

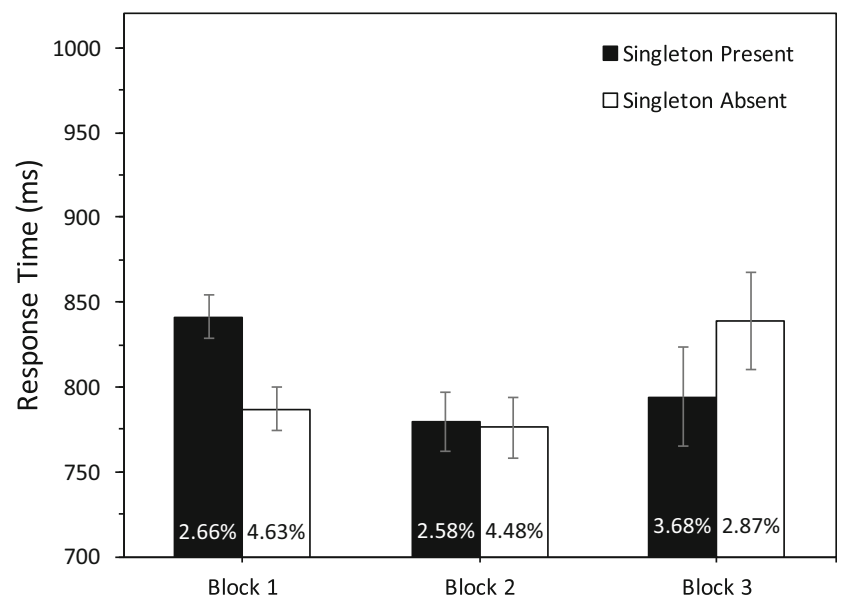

(in milliseconds) as a function of block (Blocks 1, 2, \& 3) and singleton presence (singleton present vs. singleton absent). Error rates from each condition appear in the base of each bar. Error bars represent $95 \%$ withinsubject confidence intervals (Loftus \& Masson, 1994; Baguley, 2012)

half vs. second), singleton presence (present vs. absent) and stimulus environment (Blocked vs. Mixed group). Block 4 RTs from both groups appear in Fig. 3. There was neither a main effect of trial position, $F<1$, nor singleton presence, $F<$ 1 . There was no main effect of group, $F(1,30)=1.86, p>0.18$, $\eta_{\mathrm{p}}{ }^{2}=0.26$. The ANOVA also found neither an interaction between trial position and group, $F(1,30)=2.58, p>0.1$, $\eta_{\mathrm{p}}{ }^{2}=0.08$, nor an interaction between trial position and singleton presence, $F(1,30)=1.43, p>0.24, \eta_{\mathrm{p}}{ }^{2}=0.05$. The ANOVA found a marginally significant interaction between singleton presence and group, $F(1,30)=3.92, p=0.057, \eta_{\mathrm{p}}{ }^{2}=$ 0.12 . Importantly, the results revealed a significant three-way interaction between trial position, singleton presence, and group, $F(1,30)=5.14, p<0.05, \eta_{\mathrm{p}}{ }^{2}=0.15$. This interaction demonstrates that color-singletons had a different effect on RTs depending on block half and group. Planned comparisons found that observers in the Blocked group responded slower when the color singleton was present $(962 \mathrm{~ms})$ in the first half of block 4 than when it was absent $(850 \mathrm{~ms}), t(15)=2.59, p<$ $0.05, \mathrm{~d}_{\mathrm{z}}=0.65$. Observers in the Blocked group did not respond slower when the color singleton was present $(858 \mathrm{~ms}$; singleton absent: $868 \mathrm{~ms}$ ) in the second half of the block, $t<1$. Observers in the Mixed group did not respond slower when the color-singleton was present $(773 \mathrm{~ms}$; singleton absent: 814 $\mathrm{ms})$ in the first half of block $4, t(15)=1.62, p>0.1, \mathrm{~d}_{\mathrm{z}}=0.41$. Color singletons also did not slow Mixed-group responses during the second half of the block, $t<1$ (singleton present: $804 \mathrm{~ms}$; singleton absent: $808 \mathrm{~ms}$ ).

Importantly, color singletons slowed the Blocked group's responses more in the first half of block 4 than the Mixed group's responses, $t(30)=3.05, p<0.01, \mathrm{~d}_{\mathrm{z}}=0.54$. This was not true during the second half of block $4, t<1$. These tests indicated that color singletons initially distracted 


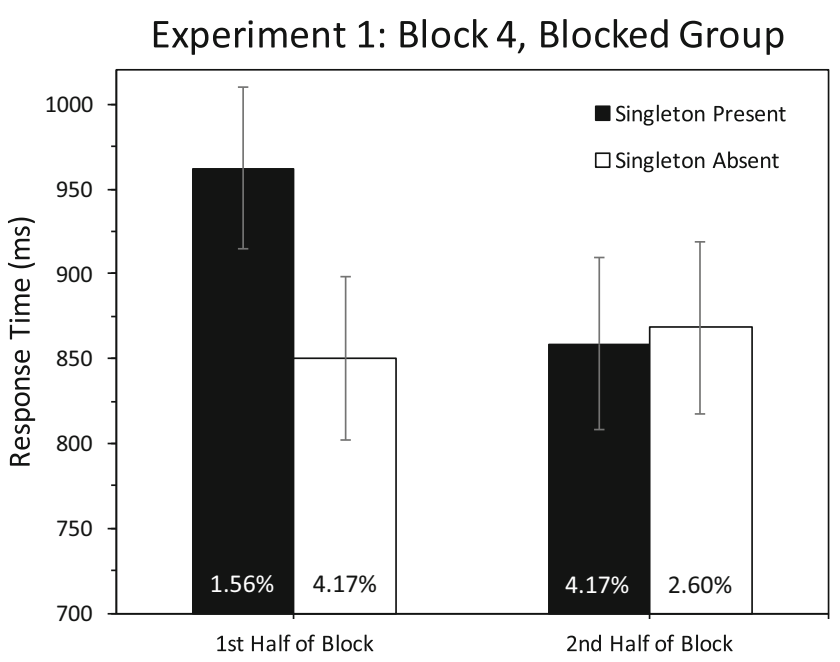

Fig. 3 Results from Block 4 of Experiment 1. The upper panel depicts RTs from block 4 of the Blocked group as a function of trial position (first half of the block vs. second half of the block) and singleton presence (singleton present vs. absent). The lower panel depicts RTs from Block

observers in the Blocked group, but not the Mixed group. Thus, a heterogeneous stimulus environment gave observers the ability to ignore a salient color-singleton distractor without previously experiencing this item.

Error rates from each condition appear in the base of the bars depicting RTs from that condition (Figs. 2 and 3). We transformed accuracy values via an arcsine square-root transformation (Freeman \& Tukey, 1950), and submitted these values to the same ANOVAs as the RTs. First, we submitted the transformed accuracy values from blocks 1-3 of the Blocked group to a $2 \times 2$ repeated measures ANOVA with the factors trial position and singleton presence. The ANOVA found neither significant main effects nor interactions, all $F_{\mathrm{S}}<1$. Next, we submitted the transformed accuracy values from blocks $1-3$ of the Mixed group to a $3 \times 2$ repeated measures ANOVA with the factors block and singleton presence. The ANOVA found neither a main effect of block, $F<1$, nor a main effect of singleton presence, $F(1,15)=2.14, p>$ $0.16, \eta_{\mathrm{p}}{ }^{2}=0.12$. The ANOVA also failed to find an interaction between block and singleton presence, $F(2,30)=1.57, p>$ $0.22, \eta_{\mathrm{p}}^{2}=0.09$. Finally, we submitted the block 4 transformed accuracy values to a $2 \times 2 \times 2$ mixed measures ANOVA with the factors trial position, singleton presence, and group. The ANOVA found no main effect of trial position, $\mathrm{F}(1,30)=2.60, p>0.11, \eta_{\mathrm{p}}{ }^{2}=0.08$, nor a main effect of singleton presence, $F<1$. The ANOVA did find a significant interaction between singleton presence, trial position, and group, $F(1,30)=9.45, p<0.005, \eta_{\mathrm{p}}^{2}=0.24$. Follow-up comparisons indicate that higher accuracy in singleton present than singleton absent trials of the blocked group during the first half of the block drove this interaction, $t(15)=2.61, p<$ $0.05, \mathrm{~d}_{\mathrm{z}}=0.65$. It is likely that the response-terminated displays kept observers' accuracy at ceiling, which hindered our

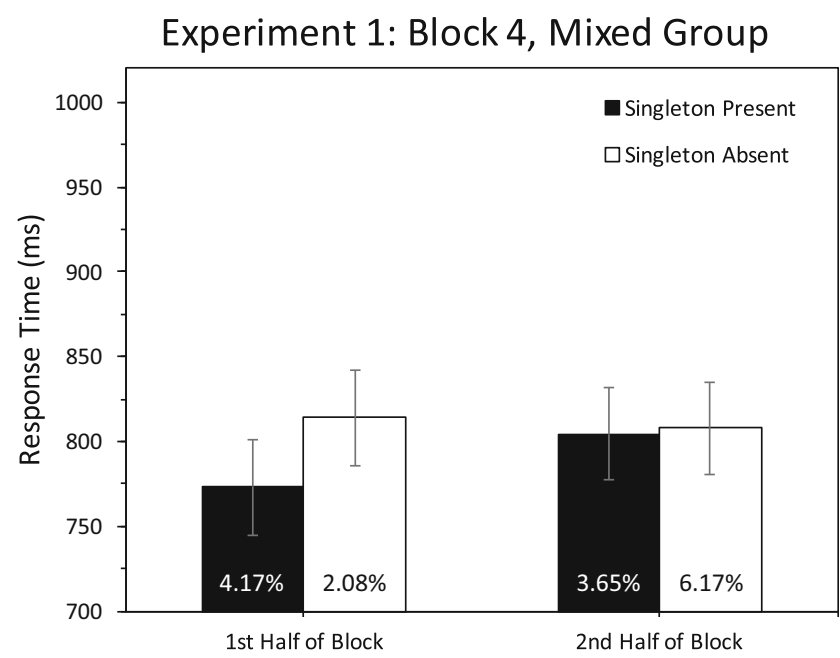

4 of the Mixed group as a function of trial position and singleton presence. Error rates appear in the base of the bars. Error bars represent 95\% within-subject confidence intervals (Loftus \& Masson, 1994; Baguley, 2012)

ability to find significant accuracy differences across the conditions.

\section{Discussion}

In Experiment 1, experience with heterogeneous salient distractors enables observers to ignore novel salient distractors (the mixed task environment), whereas experience with homogeneous salient distractors does not (the blocked task environment). Thus, learned distractor rejection obeys at least one principle observed in skill learning: Heterogeneous practice encourages generalization of learning (Schmidt \& Bjork, 1992).

The generalization observed in this experiment reflects better discrimination of targets from distractors. Discrimination requires constructing a template of both targets and distractors. In Experiment 1, experience might have encouraged a more precise target template (Bacon \& Egeth, 1994). For instance, observers might have learned to reject distractors not by learning to ignore a specific color value but by narrowing their target template (Becker, Folk, \& Remington, 2010). In our experiment, observers might begin by searching for a circle of any color and narrow their target template when experiencing salient color singleton distractors. The increased variability in the heterogeneous stimulus environment might encourage more drastic narrowing of this target template. Observers might learn to reject distractors, irrespective of the precision of the target template. The heterogeneous stimulus environment might encourage observers to create more generalizable distractor rejection templates (Arita, Carlisle, \& Woodman, 2012; also see Beck \& Hollingworth, 2015; Cunningham \& Egeth, 2016). For instance, observers might learn to reject specific distractor colors, but the heterogeneous 
stimulus environment causes observers to create less specific distractor rejection templates. These less precise distractor rejection templates make novel distractors more likely to fall within the color range specified by the rejection templates and leave observers immune to distraction by novel distractors.

These results indicate that we do not simply instantiate an attentional control state by selecting a search mode (Bacon \& Egeth, 1994) or by choosing a target template (Desimone \& Duncan, 1995). Instead, attentional control is gradually honed across experience (e.g., Logan, 2002; Chun \& Jiang, 2003; Cosman \& Vecera, 2014). The attentional control system should not be thought of as a monolithic cognitive system ruled by either stimulus-driven or goal-driven inputs. Instead, the attentional control system constantly adapts to the task, changing the priorities of stimulus and goal-driven inputs (Vecera et al., 2014). In retrospect, it seems necessary that any adaptation to a stimulus environment cannot be instantaneous, because experience over a sequence of trials is required to learn statistics of the stimulus environment which form the basis for target and distractor templates (Mozer \& Baldwin, 2008).

In Experiment 1, the overall distractor statistics observed by Blocked and Mixed groups were identical: For both groups, over the first three blocks, the distractor color was chosen from three alternatives with equal probability. The statistics differ only in the sequence of colors. One explanation for why generalization is better with heterogeneity is that learning is recency based (Mozer \& Baldwin, 2008), and performance in the final block of the experiment depends primarily on the statistics of the distractors in the penultimate block. If recency drives learning, one might expect it to be impossible for observers to learn a generalizable distractor rejection strategy in a blocked distractor environment.

Conflicting with this interpretation, past work from our lab (Vecera et al., 2014) found that when observers learn to ignore a particular salient distractor, they maintain this learned distractor rejection template across the experiment, even with intervening distractors. If observers retain a learned distractor rejection template over the course of the experiment and these templates have any imprecision such that a green distractor rejection template leads to the rejection of all greenish distractors, then as observers gain experience with different distractors they will be more likely to immediately reject novel distractors.

Experiment 2 was designed to resolve the apparent conflict between the result of Experiment 1, which suggests that learning is short lived, and the results of Vecera et al. (2014), which suggest that learning persists for longer, possibly for the duration of the entire experiment. Perhaps generalization may occur in principle with homogeneous environments, but there simply was not a sufficient number of homogeneous environments in Experiment 1 or a sufficient number of trials to observe generalization. To test this hypothesis, in Experiment 2, we presented a sequence of six, not four, homogeneous environments, and we extended the length of each block from 48 to 144 trials. Observers searched for a circle among heterogeneously shaped distractors over six blocks. A color singleton appeared in half of the trials, and the color of the color-singleton distractor was blocked.

If learned distractor rejection is purely recency based, then increasing the number of trials and blocks of different color singletons would not alter observers' distractor rejection. Specifically, observers would remain distracted by the appearance of a novel color singleton distractor, because that distractor had not been experienced previously. However, if distractor rejection is affected by longer-term learning, then the increased number of trials and/or blocks might allow for effective distractor rejection after a sufficient number of colorsingleton distractors had been experienced. In short, generalization might occur based on some number of distractors that had been encountered, even though those distractors were presented in a blocked fashion. Under this view, observers would not be distracted by the appearance of a novel color singleton in later blocks of the experiment.

\section{Experiment 2}

In Experiment 2, we investigated whether learned distractor rejection is purely recency-based or if learned distractor rejection persists across longer time scales. To distinguish between these hypotheses, we used the Blocked group design from Experiment 1, expanded the color-singleton distractor set from four colors to six, and extended the length of each block from 48 to 144 trials. Observers began the experiment with a 60 trial training-block as in Experiment 1, and by the end of the fourth block of trials, observers performed 636 trials searching for a constant target shape. By performing many trials, our hope was to provide observers with sufficient experience to form well specified target and distractor templates, producing a strong test of distractor rejection templates' ability to persist across the experiment. If learned distractor rejection is purely recency-based, then each time the salient color singleton changes color, the new distractor should slow observers' RTs, irrespective of the number of color singletons previously experienced. If learned distractor rejection is robust and persistent, then color singletons will become less distracting as observers progress through the experiment, even after changes in the singleton's color.

\section{Methods}

Participants Twenty-two observers completed the experiment for partial course credit. All reported normal or corrected to normal vision. 
Apparatus Experiment 2 used the same apparatus as Experiment 1.

Stimuli and procedure Experiment 2 used the same methods as in Experiment 1, except for the following changes. Observers completed 6 blocks of trials instead of 4 , which means the observers experienced two additional colorsingleton distractors ( 6 total), and the test blocks were lengthened to from 48 to 144 trials. The two additional colorsingleton colors were blue (RGB 0, 0, 255) and teal (RGB 0 , $255,255)$.

\section{Results}

We used the same data trimming techniques as in Experiment 1. This trimming eliminated less than $0.5 \%$ of the data. Four observers were excluded due to accuracy 2.5 standard deviations below the mean accuracy of all observers.

In Experiment 1, we found observers needed experience with salient distractors before they can effectively ignore these items (also see Vatterott \& Vecera, 2012). Observers in the Blocked group of Experiment 1 experienced four distinct color-singleton distractors, each in a different block. In the current experiment, we investigated whether experience with a greater number of color-singleton distractors might enable observers to immediately ignore novel colorsingleton distractors. For this reason, we compared observers' ability to immediately reject the first four colorsingleton distractors (blocks 1-4, when we previously found color-singleton distractors elicit distraction) to observers' ability to reject color-singleton distractors after the first four (blocks 5-6). In our previous demonstration of learned distractor rejection (Vatterott \& Vecera, 2012), we divided blocks in an early period (first 12 trials with the colorsingleton and first 12 trials without the color-singleton) and a late period (last 12 trials with the color-singleton and last 12 trials without the color-singleton). In the current experiment, we followed this convention and divided blocks into early trials (first 12 trials with the color-singleton and first 12 trials without the color-singleton) and late trials (last 12 trials with and without the color singleton). We used this binning in order to best replicate the early trials of our previous work, particularly the all critical early trials in which distraction effects occur.

The upper panel of Fig. 4 depicts observers' RTs across each of the six blocks. The lower panel of Fig. 4 groups observers' RTs across blocks 1-4 and blocks 5 and 6 . We grouped blocks 1-4 for comparison to Experiment 1 and to our previous findings, in which robust distraction occurred in the initial trials for four blocks (Vatterott \& Vecera, 2012). As is evident in the upper panel of Fig. 4 , the blocks 1-4 replicated our previous results by demonstrating that the early trials show a distraction effect that is absent in the late trials. In contrast, in blocks 5 and 6 there is little, if any, distraction, even though the distractors are novel colors.

To evaluate whether observers' ability to reject novel distractors changed after experiencing four novel color singletons, we conducted two complimentary analyses. First, because preliminary analyses found that RTs were similar across all blocks, we computed distractor scores by subtracting distractor absent RTs from distractor present RTs for the early and late trials in each block. We analyzed these difference scores with a $2 \times 6$ repeated measures ANOVA, with the factors of block (1-6) and trial order (early trials vs. late trials). ${ }^{2}$ Second, to allow comparisons to our previous work (Vatterott \& Vecera, 2012), we submitted raw RTs to a $2 \times 2$ $\times 2$ repeated measures ANOVA with the factors color singleton presence (present vs. absent), trial order (early trials vs. late trials), and experiment order (blocks 1-4 vs. blocks 5-6). By analyzing blocks 1-4 separately, we can ensure that these blocks replicate our previous findings.

Analysis of distraction across blocks The distractor scores for early and late trials across each block appear above the corresponding pairs of bars in the upper panel of Fig. 4. There was a main effect of block, indicating that the amount of distraction differed across the six blocks of trials, $F(5,85)=2.33, p=$ $0.05, \eta_{\mathrm{p}}{ }^{2}=0.12$. There was more distraction in blocks $1-4$ (averages of $26 \mathrm{~ms}, 72.3 \mathrm{~ms}, 19.4 \mathrm{~ms}$, and $31.1 \mathrm{~ms}$, respectively) than in blocks 5 and $6(5.8 \mathrm{~ms}$ and $4.2 \mathrm{~ms}$, respectively). There also was a main effect of trial order, $F(1,17)=5.0, p$ $=0.039, \eta_{\mathrm{p}}{ }^{2}=0.23$, with greater distraction for early trials $(40.9 \mathrm{~ms})$ than for late trials $(9.2 \mathrm{~ms})$. These two factors interacted, $F(5,85)=2.56, p=0.033, \eta_{\mathrm{p}}{ }^{2}=0.13$, indicating that the amount of distraction depended not only on trial order, as in our previous work, but also on block. In the last two blocks, there was no distraction in the early trials (Fig. 4).

We conducted two planned comparisons to understand fully this two-way interaction. We found that color singletons produced greater distraction in the early trials of blocks 1-4 than in the late trials of those same blocks, $t(17)=4.7 p<$ $0.001, \mathrm{~d}_{\mathrm{z}}=1.1$. This finding replicates those reported by Vatterott and Vecera (2012) and reflects the pattern in the lower left graph of Fig. 4. In contrast, there was no difference between the singleton distraction in the early and late trials in blocks 5 and $6, t(17)=1.4, p=0.19, \mathrm{~d}_{\mathrm{z}}=0.33$ (Fig. 4, lower right graph).

The error rates from each condition appear in the base of the bars depicting RTs from that condition. As with the RT data, we computed distractor scores by subtracting distractor absent errors from distractor present errors for the early and late trials in each block. We then arcsine transformed these distraction scores and submitted these values to the same ANOVAs as the RTs. Errors were low overall, and there were

\footnotetext{
${ }^{2}$ Thanks to an anonymous reviewer for suggesting this approach.
} 
Block 1

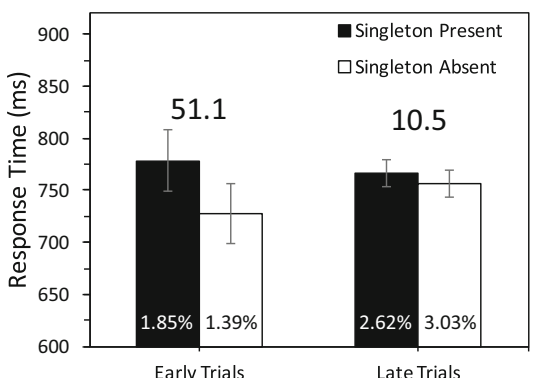

Block 4

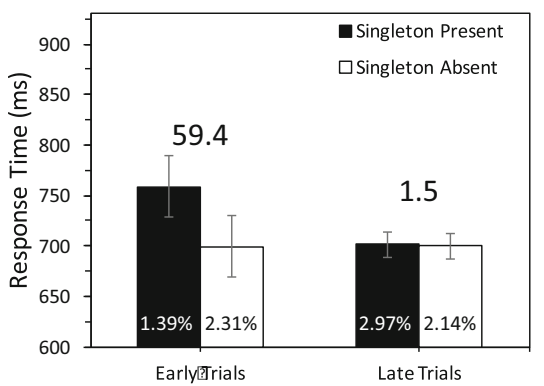

Blocks 1-4
Block 2

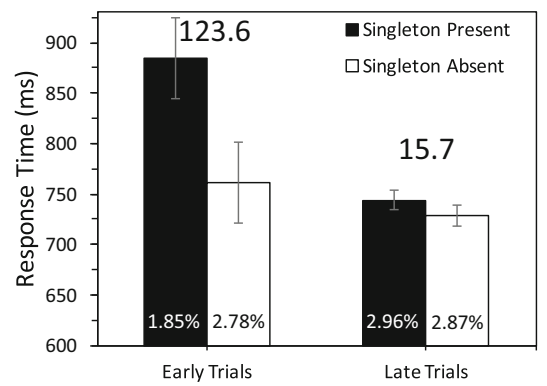

Block 5

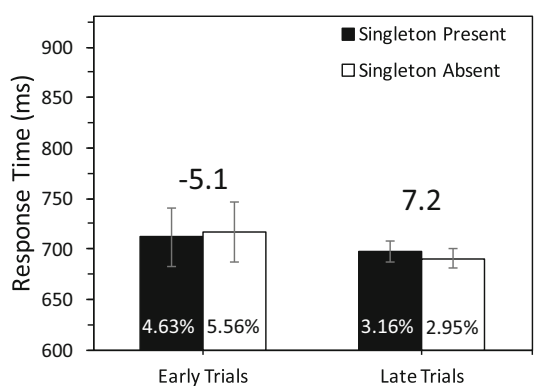

Block 3

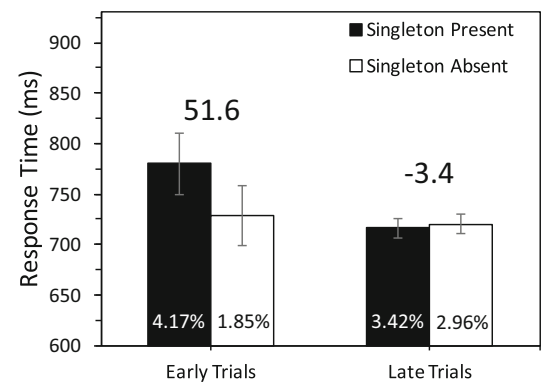

Block 6

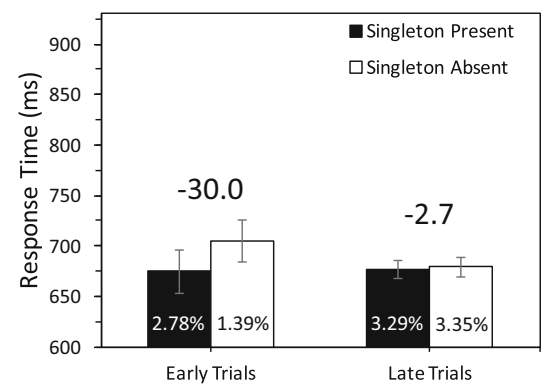

\section{Blocks 5 \& 6}

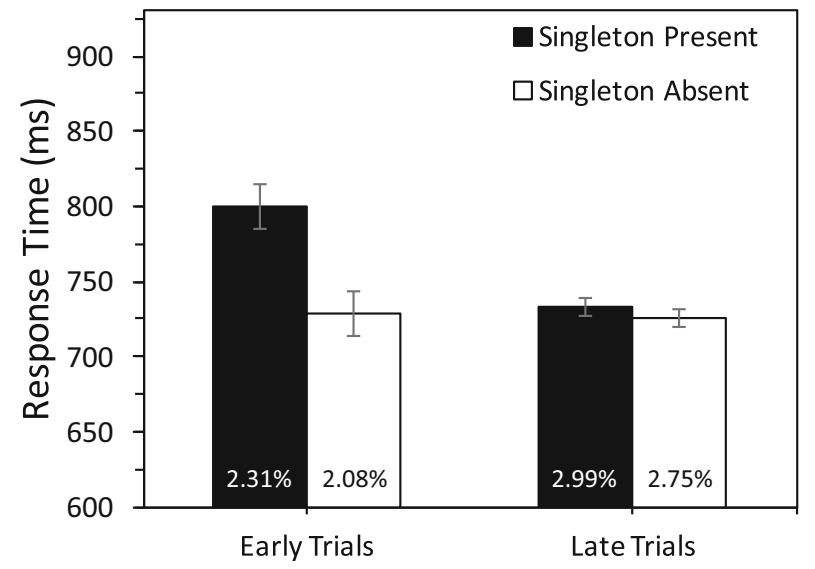

Fig. 4 Results of Experiment 2. The upper panels depict results for each block of trials as a function of trial order (early trials vs. late trials) and singleton presence (singleton present vs. singleton absent). Early trials are the first 12 trials with a color-singleton and the first 12 trials without a color-singleton. Late trials are the final 12 trials with and without a

no systematic effects in the error data. There was no main effect for block, $F(5,85)=1.8, p=0.13, \eta_{\mathrm{p}}{ }^{2}=0.09$, no main effect for trial type (early vs. late), $F(1,17)=0.39, p=0.54$, $\eta_{\mathrm{p}}^{2}=0.02$, and no interaction, $F(5,85)=0.96, p=0.45, \eta_{\mathrm{p}}{ }^{2}=$ 0.05 .

Analysis of distraction in aggregated blocks As noted, to permit comparison to our previously published results, we aggregated blocks 1-4, which matched the number of blocks in our previous work, and blocks 5-6, which provide experience with distractors beyond what we had investigated previously. We

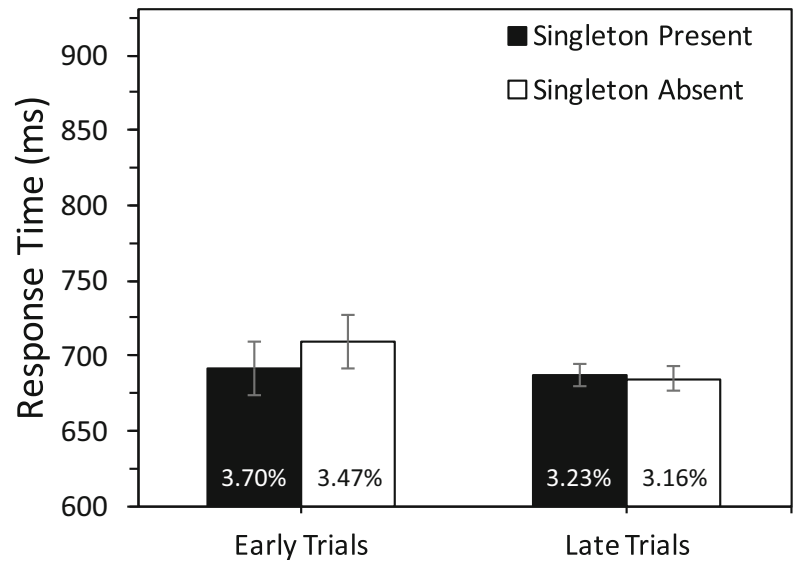

singleton distractor. The lower panels depict the results collapsed for blocks 1-4, replicating Vatterott and Vecera's (2012) analyses, and blocks 5 and 6. Error bars represent $95 \%$ within-subject confidence intervals (Loftus \& Masson, 1994; Baguley, 2012)

analyzed the RTs with a $2 \times 2 \times 2$ repeated measures ANOVA with the factors color-singleton presence (present vs. absent), trial order (early trials vs. late trials), and block order (blocks 1-4 vs. blocks 5-6). The analyses revealed an effect of singleton presence, $F(1,17)=4.22, p=0.056, \eta_{\mathrm{p}}{ }^{2}=0.20$, driven by slower RTs when the color singleton was present $(730 \mathrm{~ms})$ than absent $(711 \mathrm{~ms})$. There was marginal effect of trial order, $F(1,17)=3.0, p=0.10, \eta_{\mathrm{p}}{ }^{2}=0.15$, with slower RTs in the early trials of a block $(733 \mathrm{~ms})$ than the later trials $(708 \mathrm{~ms})$. Block order produced a significant effect, $F(1,17)=13.33, p$ $=0.002, \eta_{\mathrm{p}}{ }^{2}=0.44$, with slower RTs in the first four blocks 
(751 ms) than the last two (689 ms). We found a significant interaction between singleton presence and block order, $F(1$, $17)=7.27, p=0.015, \eta_{\mathrm{p}}{ }^{2}=0.30$. Finally, and most important, all of these effects were subsumed by a three-way interaction between singleton presence, trial order, and block order interaction, $F(1,17)=13.92, p=0.002, \eta_{\mathrm{p}}{ }^{2}=0.45$.

We conducted a series of planned comparisons to fully understand this three-way interaction. We found color singletons distracted observers in the early trials of blocks 1-4, where observers responded slower when color singletons was present $(800 \mathrm{~ms})$ than absent $(730 \mathrm{~ms}), t(17)=4.97, p<$ $0.001, \mathrm{~d}_{\mathrm{z}}=1.17$. Color singletons did not distract observers in the Late Trials of Blocks 1-4, where observers responded equally fast when the color singleton was present $(740 \mathrm{~ms})$ and absent (735 ms), $t(17)=0.64, p=0.47, \mathrm{~d}_{\mathrm{z}}=0.15$. These findings generally parallel those from the blocked group in Experiment 1 and those reported by Vatterott and Vecera (2012).

In contrast with the results from blocks 1-4, color singletons did not distract observers in blocks 5 and 6 , where observers responded equally fast when the color singleton was present (692 ms) and absent (709 ms) during the early trials, $t(17)=1.04, p=0.31, \mathrm{~d}_{\mathrm{z}}=0.25$. Similarly, there was no significant difference in RTs when the color singleton was present $(688 \mathrm{~ms})$ or absent $(669 \mathrm{~ms})$ in the late trials of blocks 5 and $6, t(17)=0.98, p=0.33, \mathrm{~d}_{\mathrm{z}}=0.23$. In blocks $1-4$, the results replicate the blocked condition of Experiment 1. In blocks 5-6, however, observers were able to reject the salient color singletons without previously experiencing them.

The error rates from each condition again appear in the base of bars in Fig. 4. We transformed accuracy values via an arcsine square-root transformation as in Experiment 1 and submitted the transformed accuracies to the same $2 \times 2 \times 2$ repeated measures ANOVA as the RTs. There were no significant effects in this analysis, all Fs $<1.8$ and $p \mathrm{~s}>0.20$.

\section{Discussion}

In Experiment 2, we found that when a novel color singleton was introduced at the beginning of blocks 1-4, observers failed to reject the color singleton effectively. However, within approximately 12 experiences with the color singleton, observers were able to reject the color singletons effectively, and color singletons ceased to distract observers, replicating our previous results (Vatterott \& Vecera, 2012) and the blocked group of Experiment 1. In the initial trials of blocks 5 and 6, a novel color singleton did not distract observers, in contrast to the initial trials of blocks 1-4. This finding suggests that learned distractor rejection is not entirely recency-based, because even in a homogeneous stimulus environment, observers can learn to reject effectively salient color singletons without previously experiencing these items. Thus, observers do retain some type of memory trace for distractor rejection that persists over the time course of the experiment, indicating that learned distractor rejection operates over multiple time scales: the short time scale of learning to suppress a novel color singleton early in blocks $1-4$, and the long time scale of learning to suppress a novel color singleton at the start of blocks 5 and 6 .

Comparing Experiment 2 to the blocked conditions of Experiment 1, we cannot determine whether the generalized distraction in blocks 5 and 6 is due to the number of distractor colors rejected in the earlier blocks of Experiment 2 or from the greater overall number of trials performed. That is, either performing more trials generally or experiencing more colorsingleton distractor identities might have led to interblock learning, which was expressed through the ability to reject immediately the novel color-singleton distractors in blocks 5 and 6. In Experiment 3, we sought to decouple these two factors by maintaining block length but limiting the number of different color-singletons in the early blocks.

\section{Experiment 3}

The number of trials and trial sequence in Experiment 3 matched that in Experiment 2, except that the color-singleton's color remained constant in blocks 1-4, limiting the number of distractor colors that observers experienced. In blocks 5 and 6, observers experienced novel color singletons, as in Experiment 2. If performing more trials in Experiment 2 relative to Experiment 1 enabled observers to reject immediately the salient color singletons, then observers should immediately reject color singletons in blocks 5 and 6 of Experiment 3 . However, if generalized distractor rejection is dependent on experiencing many different colors of singleton distractors, then color singletons should initially distract observers in blocks 5 and 6 , because observers will only have experienced one color singleton going into block 5 and two color singletons going into block 6 . Thus, the most relevant results from Experiment 3 come from blocks 5 and 6.

\section{Methods}

Participants Twenty observers completed the experiment for partial course credit. All observers reported normal or corrected-to-normal vision.

Apparatus Experiment 3 used the same apparatus as Experiments 1 and 2.

Stimuli and procedure Experiment 3 used the same methods as Experiment 2, except that the color singleton did not change color throughout blocks 1-4. Then, observers experienced new color singletons in blocks 5 and 6 . Thus, in total, observers experienced new color singletons in blocks 1,5 , and 
6. This design change resulted in only three different colorsingleton colors. Experiment 3 used red, orange, and purple as the color-singleton colors.

\section{Results}

We used the same data trimming techniques as Experiments 1 and 2. This trimming eliminated less than $0.5 \%$ of the data. We excluded two observers for mean accuracy 2.5 standard deviations below the grand average accuracy.

Figure 5 depicts observers' RTs across each of the six blocks. Because the same distractor color appeared in blocks $1-4$, blocks 1,5 , and 6 contained new distractors, and blocks 2-4 contained old (familiar) distractors. To evaluate whether observers' ability to reject novel distractors changed due to the number of trials or to the novelty/familiarity of the distractor, we first computed distractor scores by subtracting distractor absent RTs from distractor present RTs for the early and late trials in each block, as in Experiment 2. We analyzed these difference scores with a 2x6 repeated measures ANOVA, with the factors of block (1-6) and trial order (Early Trials versus Late Trials).

The distractor scores for early and late Trials across each block appear above the corresponding pairs of bars in Fig. 5. There was a main effect of block, indicating that the amount of distraction differed across the six blocks of trials, $F(5,85)=$ $3.22, p=0.01, \eta_{\mathrm{p}}{ }^{2}=0.16$. There was neither a main effect of trial type, $F(1,17)=1.82, p=0.20, \eta_{\mathrm{p}}{ }^{2}=0.10$, nor an interaction, $F(5,85)=0.98, p=0.43, \eta_{\mathrm{p}}{ }^{2}=0.06$.

The most relevant analysis of Experiment 3 comes from blocks 5 and 6 . If the absence of distraction in blocks 5 and 6 of Experiment 2 was due to the large number of trials preceding those blocks, then we should see a lack of distraction in the current experiment, because the trial numbers were identical in Experiments 2 and 3. In contrast, if the absence of distraction in Experiment 2 was due to the differently colored distractors experienced in blocks 1-4 of that experiment, then we would expect to see distraction in the current blocks 5 and 6 , because only a single distractor color had been presented in blocks 1-4 of the current experiment. There was significant distraction $(69.4 \mathrm{~ms})$ in the early trials of block $5, t(17)=2.12$, $p=0.05, \mathrm{~d}_{\mathrm{z}}=0.50$, suggesting that more than one distractor color needs to be experienced to eliminate distraction, as we observed in Experiment 2. However, the distraction in the early trials of block $6(16.5 \mathrm{~ms})$ was not significant, $t(17)=$ $1.2, p=0.24, \mathrm{~d}_{\mathrm{z}}=0.29$, suggesting that the number of trials plays some role and that generalizing distractor rejection is not determined entirely by the number of different distractors encountered.

The error rates from each condition appear in the base of the bars depicting RTs from that condition. We analyzed the error data as in Experiment 2. Errors were low overall, and there were no systematic effects in the error data. There was no main effect for block, $F(5,85)=1.35, p=0.25, \eta_{\mathrm{p}}{ }^{2}=0.07$, no main effect for trial type (early vs. late), $F(1,17)=0.45, p=$ $0.51, \eta_{\mathrm{p}}{ }^{2}=0.03$, and no interaction, $F(5,85)=0.95, p=0.45$, $\eta_{\mathrm{p}}^{2}=0.05$.

\section{Discussion}

In Experiment 2, we demonstrated that observers could learn effectively to reject novel color singletons even with previous homogeneous distractor experience. With Experiment 3, we sought to clarify whether additional task experience (i.e., the number of trials) or experience with a greater number of colorsingleton identities enabled observers to reject immediately the novel color-singleton distractors. Experiment 3 found that novel color singletons in block 5 proved highly distracting, unlike the corresponding block in Experiment 2. Had the number of trials alone determined the degree of distraction, block 5 in Experiment 3 should have found results similar to the corresponding block in Experiment 2. Instead, experience with varied distractors in Experiment 2 produced efficient distractor rejection by block 5 , and in the absence of varied distractors in the current experiment, a new distractor in a late block (block 5) proved highly distracting and slowed responses considerably.

Before entering blocks 5 , observers performed 60 practice trials and four 144 trial blocks (636 total trials). This large amount of task experience did not prevent novel color singletons from distracting observers. The lack of distraction in block 6 , however, suggests that extensive task experience also might have some role in preventing novel color singletons from distracting observers, even without experiencing many distinct colors. Although the number of different distractors encountered has a clear impact on rejecting new distractors, additional work will be necessary to disentangle the effects of the number of different distractors encountered and overall task experience, tapped by the number of trials performed by participants.

\section{General Discussion}

The experiments reported sought to examine observers' ability to reject novel, salient distractors when performing a relatively demanding visual search task. The experiments contribute to our understanding of the different factors that influence our ability to reject novel but irrelevant items. Past work has demonstrated that task strategy influences our ability to effectively reject items: searching for specific targets does achieve distractor suppression, whereas searching for unique items does not (Bacon \& Egeth, 1994; Leber \& Egeth, 2006). Previous work from our lab found observers also need itemspecific distractor experience to reject distractors effectively (Vatterott \& Vecera, 2012). The current experiments examined 
Block 1

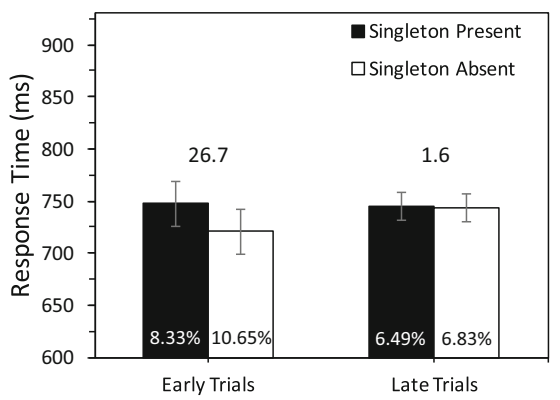

Block 4

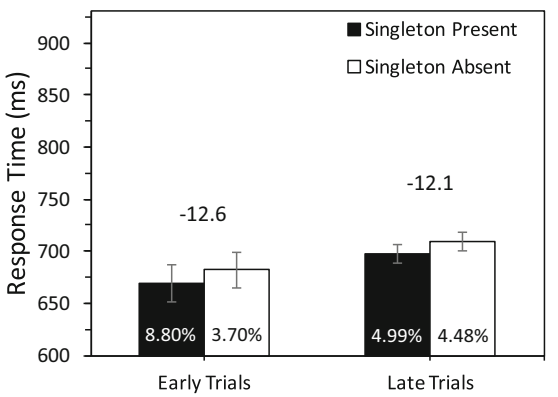

Block 2

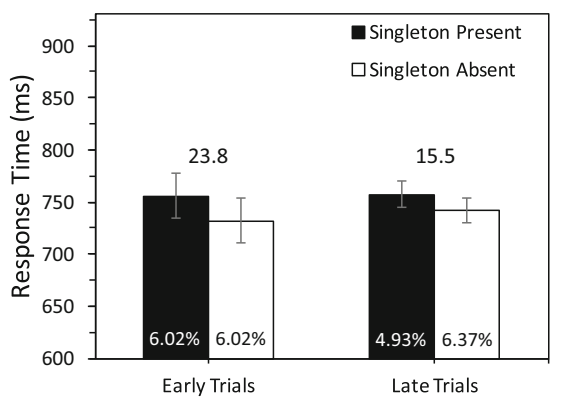

Block 5

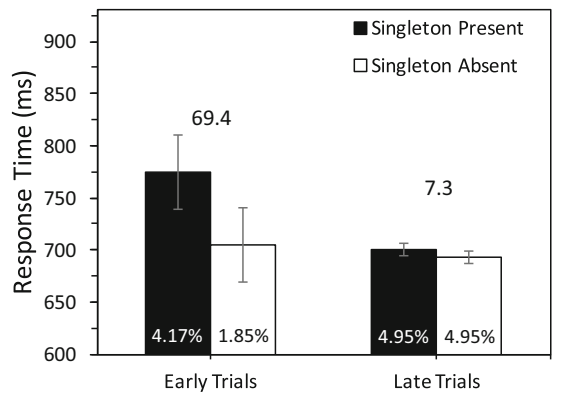

Block 3

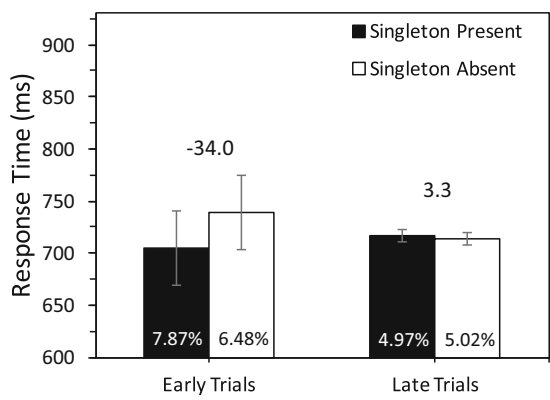

Block 6

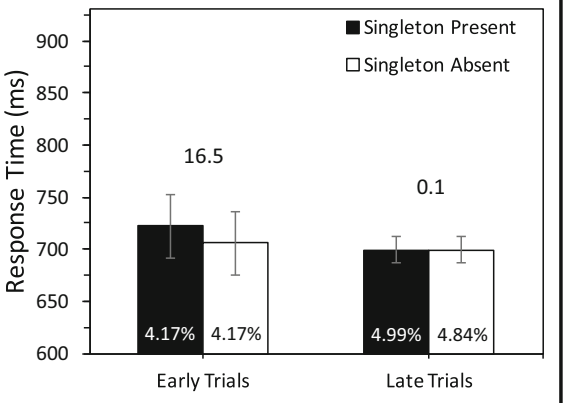

Fig. 5 Results of Experiment 3. Each graph depicts the results for a block of trials as a function of trial order (early trials vs. late trials) and singleton presence (singleton present vs. singleton absent). The distractor color is the same throughout blocks $1-4$ but changes to a new color in block 5 and

the conditions that enable observers to generalize rejection of certain distractors to novel distractors.

In Experiment 1, we examined how a heterogeneous distractor environment might enable observers to reject novel color-singleton distractors without previously experiencing these items. Previous work on learning demonstrated that heterogeneous stimulus environments encourage more generalizable skill learning (Schmidt \& Bjork, 1992). We investigated whether learned distractor rejection shares this aspect of learning. Experiment 1 found that heterogeneous distractor experience did enable observers to reject novel color-singleton distractors immediately (without practice), demonstrating that the temporal statistics of the stimulus environment is a critical factor to learning effective distractor rejection.

Experiments 2 and 3 examined the degree to which task experience and exposure to multiple singleton distractor identities is necessary for observers to immediately reject a novel color singleton distractor. Schneider and Shiffrin (1977; Shiffrin \& Schneider, 1977) found extensive experience searching for the same targets among the same distractors enabled observers to immediately guide attention to the target. Although Schneider and Shiffrin never investigated whether novel distractors could disrupt attentional guidance, their work suggests that general task experience might enable observers to effectively reject distractors without previously experiencing them. We examined this possibility in a again in block 6 . The results indicate that four blocks of extended experience with a single distractor color is insufficient to prevent distraction in block 5. Error bars represent $95 \%$ within-subject confidence intervals (Loftus \& Masson, 1994; Baguley, 2012)

homogeneous distractor environment. Experiment 2 found that observers were able to effectively reject novel color singletons following four blocks with four different distractor colors. Either general task experience or experience with many different color-singleton identities could enable observers in Experiment 2 to immediately reject novel colorsingletons. We designed Experiment 3 to distinguish between these possibilities and elucidate the conditions that enable observers to reject effectively the novel color-singleton distractors. In Experiment 3, observers performed blocks 1-4 with the same color-singleton identity and then experienced novel color singletons in blocks 5 and 6 . These novel color singletons in block 5 initially distracted observers, whereas novel color singletons in block 6 did not, indicating that experience with a greater number of color singleton identities and general task experience led to the suppression of novel color singletons in Experiment 2.

Our results appear to be consistent with a qualitative account that makes the following four key claims:

1. Each experience with a distractor yields a memory of that distractor, which will support rejection of that distractor in the future.

2. Memory of each of these experiences decays with a longtailed function of time, as has been observed for sequential learning effects (Wilder, Mozer, \& Wickens, 2011). More rapid (e.g., exponential) decay fails to explain why four single-color 
distractor blocks are insufficient to reject a novel distractor color, but five blocks is sufficient (see Experiment 2).

3. Additional experience with a given distractor color has diminishing returns, for the same reason that any type of skill learning shows a power law of learning: the individual continues to learn, but repeating an experience has a smaller effect on behavioral measures. Mozer, Colagrosso, and Huber's (2003) model provides an account by which an instancebased memory yields power law speed ups.

4. Memory generalizes in the Shepard (1980) sense to similar colors. Consequently, increasing the number of distractor colors experienced also improves the transfer to a new distractor color.

Our results have important implications for current theories of attentional control. Müller's feature dimension weighting hypothesis (Müller, Heller, \& Ziegler, 1995) claims that observers weight feature dimensions when selecting where to allocate attention. For example, when observers search for a specific shape, they place greater attentional priority on the shape dimension than color. A strict interpretation of Müller's feature dimension weighting hypothesis predicts that once observers experience one color-singleton distractor, they should bias attentional priorities away from the color dimension and effectively reject all future color-singletons, but this is not the case (Zehetleitner, Goschy, \& Müller, 2012; Vatterott \& Vecera, 2012). Instead, observers must tune attention away from multiple color values. Experiment 1 qualified this and suggests trial-to-trial heterogeneity of distractor colors guides attentional priorities away from distractor colors generally. Taken together, Experiments 2 and 3 indicate that even with trial-to-trial homogeneity, extensive experience with more than four colorsingletons also is sufficient to guide attentional prioritization away from all possible distractor colors.

Existing accounts of overcoming attentional capture can be reconciled with the current set of results. For instance, Theeuwes (1992) postulated that distractors more salient than the target always capture attention (for review see Theeuwes, 2010). Observers then redirect attention towards task-relevant items by disengaging attention from distractors. It is possible that observers increase disengagement speeds as they gain experience with salient distractor identities. The current set of results allow for experience-dependent changes in disengagement speeds. Folk and Remington (1998) proposed that salient items do not capture attention. Instead, salient distractors compete for attention, slowing the allocation of attention to the target and extending RTs. It is possible that experience with a distractor reduces salient distractors' ability to compete for attention. There also have been multiple demonstrations of priming in attentional control (Kristjánsson \& Campana, 2010; Lamy, Carmel, Egeth, \& Leber, 2006; Olivers \& Humphreys, 2003; Pinto, Olivers, \& Theeuwes, 2005). We believe our results are consistent with these rapid, experience-dependent changes in attentional control, because a novel salient distractor also is a distractor that has not been encountered in the recent past. We acknowledge, however, that the influence of a heterogeneous distractor environment and of experiencing many previous salient color singletons operates on a much longer time scale than typically seen in priming.

Horstmann's $(2002,2005)$ work on novelty and attentional capture compliments our results. In these papers, Horstmann demonstrates a novel item's power to capture observers' attention. We believe our results derive from a similar mechanism. Horstmann proposed the expectancy match hypothesis (Horstmann 2005; Becker \& Horstmann, 2011), which posits that unexpected items automatically capture observers' attention. Experiment 1 compliments Horstmann's results by demonstrating that a heterogeneous distractor environment changes observers' expectancies such that novel color singletons are no longer unexpected. Experiments 2 and 3 found that experiencing more than four color-singleton distractors has the same effect.

Baldi and Itti (2010) proposed that Bayesian expectations could determine stimulus saliency. Under this account, more unexpected events are more salient. This model improves upon other models of saliency by quantifying saliency as not only the uniqueness of an item within a single image (Itti \& Koch, 2000) but also as the uniqueness of an item across images. A model, such as Baldi and Itti's, would likely produce similar results as shown in this study. In Experiment 1, the heterogeneous environment could decrease the system's expectations of what salient distractors might appear in the future and thus create less of an expectancy violation when a novel color singleton appears. Also experiencing a multitude of different color-singleton identities might make the model less "surprised" when it experiences yet another novel color singleton. However, purely rational accounts, such as Baldi and Itti (2010), have a challenge in explaining the volume of practice that is needed for altering expectations (Experiments 2 and 3 vs. Experiment 1) and the effects of sequence ordering (Experiment 1).

In addition to learning distractor rejection based on mere exposure as in the current studies, distractors can be rejected by explicitly directing (cuing) observers to an upcoming distractor (Cunningham \& Egeth, 2016; Moher \& Egeth, 2012). In early trials of an experiment, instructing observers with a cue, such as the words "ignore red," initially draws attention to those cued items and slows responses over a neutral cue baseline. However, after several hundred trials, observers learn to reject an item based on an explicit cue and find targets faster when cued what to ignore than when given a neutral cue (Cunningham \& Egeth, 2016). This cued distractor rejection is learned if one distractor color, such as red, is cued throughout the experiment; if many, variable distractors are cued ("ignore red" on one trial, "ignore blue" later, etc.), observers are unable to learn cued distractor rejection, and responses are no faster following ignore cues than following neutral cues. This latter finding appears at odds with the 
current results, in which we demonstrate learning to reject multiple, varying distractors (most directly in Experiment 1). There are numerous differences between our task and Cunningham and Egeth's (2016), which complicate a direct comparison of the results. Perhaps most relevant, our participants learned to reject three distractor colors, but Cunningham and Egeth (2016) cued eight different distractor colors. The apparent discrepancy between the two sets of findings might suggest that only a small number of distractors can learn to be rejected. However, we hypothesize that the inability to learn to reject multiple distractors is caused by the explicit cuing itself. Explicit cuing not only draws attention initially to distractors, but cues also serve as a "prompt" that may refresh attention to the cued item and therefore delay learned distractor rejection. We are currently exploring the similarities and differences between cued distractor rejection and the uncued, implicit learned rejection we have reported here and elsewhere. Similar mechanisms might underlie both cued and implicit distractor rejection once the initial attraction to a cued item is overcome.

Finally, our results combine with the growing literature demonstrating that experience plays a substantial role in attentional control. Each experiment presented adds important understanding to this literature. For instance, Experiment 1 demonstrates the role of learning context in experience's influence on attentional control. Experiments 2 and 3 specify how distractor experience influences attentional control. Specifically, experiencing a multitude of different salient distractor identities encourages observers to strengthen goaldriven inputs to attentional control. This demonstration supports the idea we have promoted in past work (Wilder et al., 2011; Vecera et al., 2014) that attentional control is not a fixed system that always biases attention towards either salient or task-relevant items, which tends to be the historically dominant view in the literature. Instead, the attentional control system is always in a state of flux, constantly adjusting to maximize success, whether success is defined by a reward (Anderson, Laurent, \& Yantis, 2011) or by intrinsic measures, such as efficiency in discriminating targets from distractors.

Acknowledgements This research was supported in part by grants from the National Science Foundation (BCS 11-51209) and by research contracts from the Nissan Motor Corporation and the Toyota Collaborative Safety Research Center (CSRC).

\section{References}

Anderson, B.A., Laurent, P.A., \& Yantis, S. (2011). Value-driven attentional capture. Proceedings of the National Academy of Sciences, 108(25), 10367-10371.

Arita, J.T., Carlisle, N.B., \& Woodman, G.F. (2012). Templates for rejection: Configuring attention to ignore task-irrelevant features.
Journal of Experimental Psychology: Human Perception and Performance, 38(3), 580-584.

Awh, E., Belopolsky, A.V., \& Theeuwes, J. (2012). Goal-driven versus stimulus-driven attentional control: A failed theoretical dichotomy. Trends in Cognitive Sciences, 16(8), 437-443.

Bacon, W. F., \& Egeth, H. E. (1994). Overriding stimulus-driven attentional capture. Perception \& Psychophysics, 55, 485-96.

Baguley, T. (2012). Calculating and graphing within-subject confidence intervals for ANOVA. Behavior Research Methods, 44(1), 158-175.

Baldi, P., \& Itti, L. (2010). Of bits and wows: A Bayesian theory of surprise with applications to attention. Neural Networks, 23, 649666.

Beck, V. M., \& Hollingworth, A. (2015). Evidence for negative feature guidance in visual search is explained by spatial recoding. Journal of Experimental Psychology: Human Perception and Performance, 41(5), 1190-1196. https://doi.org/10.1037/xhp0000109

Becker, S.I., Folk, C.L., \& Remington, R.W. (2010). The role of relational information in contingent capture. Journal of Experimental Psychology: Human Perception and Performance, 36, 1460-1476.

Becker, S.I., \& Horstmann, G. (2011). Novelty and saliency in attentional capture by unannounced motion singletons. Acta Psychologica, 136(3), 290-299.

Brainard, D.H. (1997). The psychophysics toolbox. Spatial Vision, 10(4), 433-464.

Catalano, J.F., \& Kleiner, B.M. (1984). Distant transfer in coincident timing as a function of variability of practice. Perceptual and Motor Skills, 58, 851-856.

Chun, M.M. (2000). Contextual cueing of visual attention. Trends in Cognitive Sciences, 4(5), 170-178.

Chun, M.M., \& Jiang, Y. (1998). Contextual cueing: implicit learning and memory of visual context guides spatial attention. Cognitive Psychology, 36(1), 28-71.

Chun, M.M., \& Jiang, Y. (2003). Implicit, Long-Term Spatial Contextual Memory. Journal of Experimental Psychology: Learning, Memory, and Cognition, 29(2), 224-234.

Cosman, J.D., \& Vecera, S.P. (2014). Establishment of an attentional control setting via statistical learning. Journal of Experimental Psychology: Human Perception and Performance, 40(1), 1-6.

Cunningham, C. A., \& Egeth, H. E. (2016). Taming the white bear: Initial costs and eventual benefits of distractor inhibition. Psychological Science, 27(4), 476-485.

Desimone, R., \& Duncan, J. (1995). Neural mechanisms of selective visual attention. Annual Review of Neuroscience, 18, 192-222.

Folk, C. L., Remington, R. W. (1998). Selectivity in distraction by irrelevant featural singletons: Evidence for two forms of attentional capture. Journal of Experimental Psychology: Human Perception and Performance, 24, 847-858.

Folk, C. L., Remington, R. W., \& Johnston, J. C. (1992). Involuntary covert orienting is contingent on attentional control settings. Journal of Experimental Psychology: Human Perception and Performance, 18(4), 1030-44.

Freeman, M.F., \& Tukey, J. (1950). Transformations related to the angular and the square root. The Annals of Mathematical Statistics, 21, 607611.

Gaspelin, N., Leonard, C. J., \& Luck, S. J. (2017). Suppression of overt attentional capture by salient-but-irrelevant color singletons. Attention, Perception, \& Psychophysics, 79(1), 45-62.

Gaspelin, N., Leonard, C. J., \& Luck, S. J. (2015). Direct evidence for active suppression of salient-but-irrelevant sensory inputs. Psychological Science, 26(11), 1740-1750.

Homa, D., \& Vosburgh, R. (1976). Category breadth and the abstraction of prototypical information. Journal of Experimental Psychology: Human Learning and Memory, 2(3), 322-330. https://doi.org/10. 1037/0278-7393.2.3.322 
Horstmann, G. (2002). Evidence for attentional capture by a surprising color-singleton in visual search. Psychological science, 13(6), 499505 .

Horstmann, G. (2005). Attentional capture by an unannounced colorsingleton depends on expectation discrepancy. Journal of Experimental Psychology: Human Perception and Performance, 31, 1039-1060.

Itti, L., \& Koch, C. (2000). A saliency-based search mechanism for overt and covert shifts of visual attention. Vision Research, 40, 14891506.

Kerr, R., \& Booth, B. (1978). Specific and varied practice of motor skill. Perceptual and Motor Skills, 46(2), 395-401. https://doi.org/10. 1177/003151257804600201

Kristjánsson, Á., \& Campana, G. (2010). Where perception meets memory: A review of repetition priming in visual search tasks. Attention, Perception, \& Psychophysics, 72(1), 5-18. https://doi.org/10.3758/ APP

Lamy, D., Carmel, T., Egeth, H., \& Leber, A. (2006). Effects of search mode and inter-trial priming on singleton search. Perception \& Psychophysics, 68, 919-932.

Leber, A. B., \& Egeth, H. E. (2006). It's under control: goal-driven search strategies can override attentional capture. Psychonomic Bulletin \& Review, 13(1), 132-8.

Lively, S. E., Logan, J. S., \& Pisoni, D. B. (1993). Training Japanese listeners to identify English/r/and/1/. II: The role of phonetic environment and talker variability in learning new perceptual categories. The Journal of the Acoustical Society of America, 94(3), 1242-1255. https://doi.org/10.1121/1.408177

Loftus, G. R., \& Masson, M. E. J. (1994). Using confidence intervals in within-subject designs. Psychonomic Bulletin \& Review, 1, 476490

Logan, G. D. (2002). An instance theory of attention and memory. Psychological Review, 109(2), 376-400.

Maljkovic, V., \& Nakayama, K. (1994). Priming of pop-out: I. Role of features. Memory \& Cognition, 22(6), 657-672. https://doi.org/10. 3758/BF03209251

Maljkovic, V., \& Nakayama, K. (1996). Priming of pop-out: II. The role of position. Perception \& Psychophysics, 58(7), 977-991. https:// doi.org/10.3758/BF03206826

Maljkovic, V., \& Nakayama, K. (2000). Priming of popout: III. A shortterm implicit memory system beneficial for rapid target selection. Visual Cognition, 7(5), 571-595. https://doi.org/10.1080/ 135062800407202

Moher, J., \& Egeth, H. E. (2012). The ignoring paradox: Cueing distractor features leads first to selection, then to inhibition of tobe-ignored items. Attention, Perception, \& Psychophysics, 74(8), 1590-1605. https://doi.org/10.3758/s13414-012-0358-0

Mozer, M. C., \& Baldwin, D. (2008). Experience-guided search: A theory of attentional control. In J. Platt, D. Koller, \& Y. Singer (Eds.),
Advances in neural information processing systems, 20 (pp. 10331040). Cambridge: MIT Press.

Mozer, M. C., Colagrosso, M. D., \& Huber, D. E. (2003). Mechanisms of long-term repetition priming and skill refinement: A probabilistic pathway model. In R. Alterman \& D. Kirsh (Eds.), Proceedings of the Twenty Fifth Annual Conference of the Cognitive Science Society (pp. 828-833). Boston: Cognitive Science Society.

Müller, H.J., Heller, D., \& Ziegler, J. (1995). Visual search for singleton feature targets within and across feature dimensions. Perception \& Psychophysics, 57(1), 1-17.

Olivers, C. N., \& Humphreys, G. W. (2003). Attentional guidance by salient feature singletons depends on intertrial contingencies. Journal of Experimental Psychology: Human Perception and Performance, 29, 307-318.

Pashler, H. (1988). Cross-dimensional interaction and texture segregation. Perception \& Psychophysics, 43, 307-318.

Pinto, Y., Olivers, C. N. L., \& Theeuwes, J. (2005). Target uncertainty does not lead to more distraction by singletons: Intertrial priming does. Perception \& Psychophysics, 67(8), 1354-61.

Schmidt, R.A., \& Bjork, R.A. (1992). New conceptualizations of practice: Common principles in three paradigms suggest new concepts for training. Psychological Science, 3(4), 207-217.

Schneider, W., \& Shiffrin, R.M. (1977). Controlled and automatic human information processing: I. Detection, search, and attention. Psychological Review, 84(1), 1-66.

Shepard, R. N. (1980). Multidimensional scaling, tree-fitting, and clustering. Science, 210(4468), 390-398.

Shiffrin, R.M., \& Schneider, W. (1977). Controlled and automatic human information processing: II. Perceptual learning, automatic attending, and a general theory. Psychological Review, 84(2), 127-190.

Theeuwes, J. (1992). Perceptual selectivity for color and form. Perception \& Psychophysics, 51, 599-606.

Theeuwes, J. (2010). Goal-driven and stimulus-driven control of visual selection. Acta Psychologica, 135(2), 77-99.

Vatterott, D. B. (2015). Learning to overcome distraction (Doctoral Dissertation). University of Iowa.

Vatterott, D.B., \& Vecera, S.P. (2012). Experience-dependent attentional tuning of distractor rejection. Psychonomic Bulletin \& Review, 19(5), 871-878.

Vecera, S. P., Cosman, J. D., Vatterott, D. B., \& Roper, Z. J. J. (2014). The control of visual attention: Toward a unified account. In Brian $\mathrm{H}$. Ross (Ed.), Psychology of learning and motivation (Vol. 60, pp. 303-347). Academic Press.

Wilder, M.H., Mozer, M.C., \& Wickens, C.D. (2011). An integrative, experience-based theory of attentional control. Journal of Vision, 11(2), 8 .

Zehetleitner, M., Goschy, H., Müller, H.J. (2012). Goal-driven control of attention: It's gradual, practice-dependent, and hierarchically organized. Journal of Experimental Psychology: Human Perception and Performance, 38(4), 941-957. 